\title{
Polymer depletion interaction of small mesoscopic particles: Effects beyond leading order and anisotropic particles
}

\author{
E. Eisenriegler, ${ }^{\text {a) }}$ A. Bringer, and R. Maassen \\ Institut für Festkörperforschung, Forschungszentrum Jülich, D-52425 Jülich, Germany
}

(Received 16 January 2003; accepted 7 February 2003)

\begin{abstract}
We discuss the depletion interaction between a wall and a mesoscopic particle of ellipsoidal shape induced by long, flexible, nonadsorbing polymer chains. Both a force and a torque are exerted on the particle. We concentrate on the case in which the particle size is much smaller than typical polymer lengths, such as the radius of gyration $\mathcal{R}_{g}$, where a rigid polymer approximation of the AsakuraOosawa-type cannot be applied. Explicit analytical results are obtained for ideal polymers. For particle-wall distances $z$ large compared to $\mathcal{R}_{g}$ an orientation of the ellipsoid perpendicular to the wall is favored. For $z$ small compared to $\mathcal{R}_{g}$ (but $z$ still large compared to the particle size), parallel orientation is favored. The perturbation of the polymer system due to the small particle is represented by a series of point-operators in the corresponding field theory, with next-to-next-to-leading anisotropic derivative-operators characterizing the particle orientation. For the interaction between a spherical particle and a wall the simple analytical results predicted by the proposed small particle expansion beyond leading order display an interesting structure which is confirmed by direct numerical computation. (C) 2003 American Institute of Physics.
\end{abstract}

[DOI: $10.1063 / 1.1565101]$

\section{INTRODUCTION}

Colloid science covers a broad class of substances encompassing milk, blood, and paints. A major goal is to understand the effective interactions between mesoscopic colloidal particles. These can be tuned in various ways by manipulating the solvent. One way is by adding nonadsorbing free polymer chains. For entropic reasons nonadsorbing chains avoid the space between two particles, leading to an unbalanced pressure which pushes the two particles towards each other. Depletion forces for an isolated pair of immersed particles or for a single immersed particle near a wall were measured in recent experiments. ${ }^{1-3}$ The polymer depletion interaction is also of relevance for the separation of proteins. $^{4-6}$

The polymer depletion interaction depends on the degree of inter-chain overlap and on the size ratio between the colloidal particle and the polymer chains. For a dilute solution of polymer chains with radius of gyration $\mathcal{R}_{g}$ much smaller than the particle size, a qualitatively correct description is obtained by viewing the polymer coils as nondeformable hard spheres, ${ }^{7}$ with a radius of the order of $\mathcal{R}_{g}$. This approach has been applied both to large spherical colloidal particles and to anisotropic particles such as disks or platelets. ${ }^{8,9}$ However, for particle size much smaller than $\mathcal{R}_{g}$ (but much larger than the polymer persistence length), polymer conformations coiling around the particle are important, and the above Asakura-Oosawa treatment does not apply.

Polymer depletion near small mesoscopic particles is of interest for protein solutions and has first been discussed by de Gennes. ${ }^{10}$ Odijk has given a mean-field discussion for spherical $^{11}$ and ellipsoidal ${ }^{12}$ small particles in a semidilute

${ }^{\text {a)} E l e c t r o n i c ~ m a i l: ~ e . e i s e n r i e g l e r @ f z-j u e l i c h . d e ~}$ polymer solution. Recent computer simulations investigated the effective depletion interaction between small spherical particles $^{13}$ or between a sphere and a wall ${ }^{14}$ in the presence of the excluded volume interaction between chain monomers. In Refs. 15-18 it was pointed out that the perturbation of the polymer system due to a small spherical particle can, in leading order, be viewed as a $\delta$ function potential repelling the chain-monomers with an amplitude that equals a universal number times the particle radius raised to a universal exponent. The exponent is ${ }^{10}$ the scaling dimension $d-1 / \nu$ of the monomer density with $d$ the dimension of space and $\nu$ the Flory exponent. This is an operator relationship similar in spirit to operator-product expansions in field theory. ${ }^{19}$ Since the amplitude is independent of distant perturbations, such as other particles or a wall, the same power of the radius and the same universal number appear in the free energy of immersing a particle in bulk polymer solution and in the effective interaction between two particles or between a particle and a wall. Moreover, replacing the mesoscopic particle acting via boundary conditions on the polymer system by a simple density operator considerably simplifies the evaluation of these interactions, by relating them to the densitydensity correlation function in bulk polymer solution or to the density profile in a polymer solution in the half space bounded by a wall. ${ }^{17,18}$ Similar point-operator representations or "small particle expansions" have proven useful in other examples of boundary critical phenomena, such as mesoscopic particles immersed in a critical fluid mixture. ${ }^{20-23}$ For a discussion of the close relation between long flexible polymer chains and critical behavior, in particular, between polymers near surfaces and boundary critical phenomena, see Refs. 24-27, respectively. Boundary critical phenomena are reviewed in Refs. 28 and 29. 

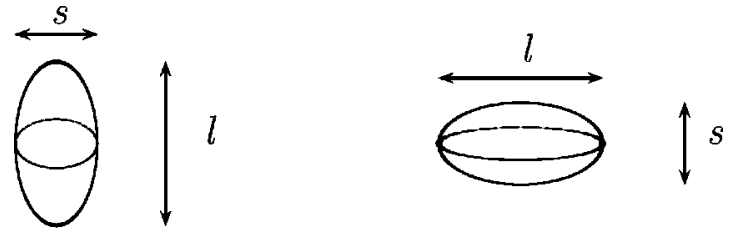

cigar

pancake

$s<<l:$

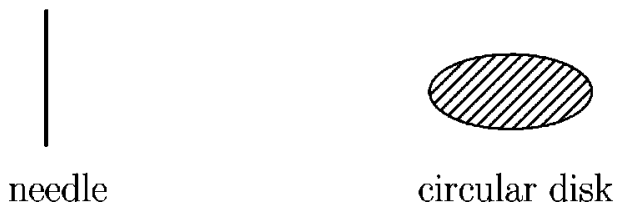

FIG. 1. Prolate (cigar-shaped) and oblate (pancake-shaped) ellipsoids of revolution with long axis $l$ and short axis $s$. For $s \ll l$ the cigar reduces to a needle and the pancake to a circular disk.

In the present paper we extend the small particle expansion to anisotropic particles of ellipsoidal shape in a polymer solution. The anisotropic effects of small particles, such as the prolate or oblate ellipsoids of revolution shown in Fig. 1, are related to operators containing anisotropic spatial derivatives. These have a higher scaling dimension than the monomer density operator, which is isotropic, and thus are accompanied by the particle size raised to a greater power than the exponent $d-1 / \nu$ of de Gennes. In order to consider anisotropy, the small particle expansion must be extended beyond the leading order.

Here we study the simplest case of an ellipsoidal particle in a solution of ideal polymers. This is a first step toward the more realistic case of "real" polymers with excluded volume interaction between chain monomers or the marginal behavior with "logarithmic corrections" of polymers at the theta point. ${ }^{24-27}$ As we shall see, for ideal nonadsorbing polymers the scaling dimension of the leading anisotropic operators is $d$, and thus the anisotropic effects of, e.g., a small circular disk in three dimensions, are proportional to the third power of the radius of the disk. There are also isotropic operators beyond the leading density operator. The dominant operators are the next-to-leading operator with a scaling dimension $2(d-2)$, a higher operator with dimension $3(d-2)$, and two others with dimension $d$. Thus in $d=3$ there are three next-to-next-to-leading isotropic operators of scaling dimension 3. These higher isotropic operators survive, even in the special case of a spherical particle, in which the amplitudes of the anisotropic operators vanish, and describe corrections in the physical properties which are of higher order in the radius of the sphere.

In Sec. II we first consider a single spherical particle and determine the above-mentioned higher isotropic operators and their amplitudes. In addition we find a "contact term" between the polymer ends and the particle. Corresponding terms are also known from operator-product expansions. ${ }^{19}$ From this we then predict, in three dimensions, the leading, next-to-leading, and next-to-next-to-leading contributions to the polymer-induced interaction between the small sphere and a wall which are of order $R, R^{2}$, and $R^{3}$, respectively, in the sphere radius $R$. The predictions are compared with the numerical calculation of the interaction for arbitrary size ratio $R / \mathcal{R}_{g}$ in Ref. 17. Since each order of the interaction involves a different nontrivial functional dependence on $z_{S} / \mathcal{R}_{g}$, with $z_{S}$ the particle-wall distance, this is an interesting and important check of the operator expansion. The expansion yields analytic expressions for the numerical results ${ }^{17}$ of the higher order distance-dependencies of the particle-wall interaction. Finally we consider the interaction between two spheres ${ }^{30}$ and calculate the next-to-leading contribution for small particle radii.

In Sec. III we discuss the expansion for small prolate and oblate ellipsoidal particles. Again we determine operators and amplitudes from single-particle properties and we find that the particle-wall interaction depends on both the particle-wall distance $z_{E}$ and the orientation of the particle with respect to the wall. Interestingly, the preferred orientation changes from perpendicular to parallel to the wall on decreasing $z_{E}$ from large to small values compared to the polymer size $\mathcal{R}_{g}$.

\section{DEPLETION EFFECTS FOR A SMALL SPHERICAL PARTICLE BEYOND LEADING ORDER}

Here we consider mesoscopic particles of spherical shape. Besides a single spherical particle interacting with a nonadsorbing ideal polymer chain, we discuss the polymerinduced depletion interaction between a particle and a wall and between two particles and generalize previous results beyond leading order in the particle size. This also serves to introduce the "small particle expansion" in its simplest form.

A basic quantity in the conformational statistics of ideal polymer chains is the partition function $Z\left(L ; \mathbf{r}_{1}, \mathbf{r}_{2}\right)$ of a single chain $^{24}$ with the two ends fixed at $\mathbf{r}_{1}, \mathbf{r}_{2}$. Here the length of the chain, i.e., the number of monomers, is specified by the quantity

$$
L=\mathcal{R}_{e e}^{2} /(2 d)=3 \mathcal{R}_{g}^{2} / d,
$$

where $\mathcal{R}_{e e}^{2}$ is the mean-square end-to-end distance, and $\mathcal{R}_{g}^{2}$ is the mean-square radius of gyration of the fluctuating chain in the absence of boundaries.

Our goal is to determine $Z$ in the presence of impenetrable and nonadsorbing mesoscopic particles. If the polymer size $\mathcal{R}_{g}$ and the particle sizes are much larger than the polymer persistence length and the extrapolation lengths of the particle surfaces, the partition function of the randomwalk like ideal polymer satisfies ${ }^{24}$ the diffusion equation

$$
\left(\frac{\partial}{\partial L}-\Delta_{\mathbf{r}_{1}}\right) Z\left(L ; \mathbf{r}_{1}, \mathbf{r}_{2}\right)=0,
$$

where $L$ plays the role of time, with "initial condition"

$$
Z\left(L ; \mathbf{r}_{1}, \mathbf{r}_{2}\right)=\delta\left(\mathbf{r}_{1}-\mathbf{r}_{2}\right) \quad \text { for } L \rightarrow 0,
$$

and boundary conditions

$$
Z\left(L ; \mathbf{r}_{1}, \mathbf{r}_{2}\right) \rightarrow 0 \quad \text { for } \mathbf{r}_{1} \rightarrow \sigma \quad \text { or } \mathbf{r}_{2} \rightarrow \sigma,
$$

on the nonadsorbing surfaces $\sigma$ of the particles. 
The small particle expansion is stated most easily in terms of the "magnetic" (or field theory) analogue of the polymer system. ${ }^{24}$ The Laplace transform

$$
G\left(t ; \mathbf{r}_{1}, \mathbf{r}_{2}\right)=\int_{0}^{\infty} d L e^{-L t} Z\left(L ; \mathbf{r}_{1}, \mathbf{r}_{2}\right)
$$

of the ideal chain partition function $Z$ with respect to $L$, which satisfies an "Ornstein-Zernicke equation"

$$
\left(t-\Delta_{\mathbf{r}_{1}}\right) G\left(t ; \mathbf{r}_{1}, \mathbf{r}_{2}\right)=\delta\left(\mathbf{r}_{1}-\mathbf{r}_{2}\right),
$$

equals the order-parameter correlation function in a Ginzburg-Landau field theory. With the notation

$$
\varphi_{1,2}=\Phi\left(\mathbf{r}_{1}\right) \Phi\left(\mathbf{r}_{2}\right)
$$

for the product of Ginzburg-Landau fields $\Phi$,

$$
G\left(t ; \mathbf{r}_{1}, \mathbf{r}_{2}\right)=\left\langle\varphi_{1,2}\right\rangle .
$$

In the case of ideal polymers the angular brackets denote an average with a Gaussian thermal weight $\exp (-H[\Phi])$, where $H$ is the quadratic Hamiltonian

$$
H=\int d \mathbf{r}\left[\frac{1}{2}(\nabla \Phi)^{2}+\frac{t}{2} \Phi^{2}\right]+\int d \sigma \frac{c}{2} \Phi^{2} .
$$

Here $\int d \mathbf{r}$ is an integration over the volume outside the particles, i.e., the volume accessible to the polymers, and the integral $\int d \sigma$ extends over the surfaces of the particles, with the limit $c \rightarrow \infty$ taken so that $\left\langle\varphi_{1,2}\right\rangle$ vanishes if $\mathbf{r}_{1}$ or $\mathbf{r}_{2}$ approaches a particle surface.

The small particle expansion allows one to express the correlation function in the presence of a small particle in terms of correlation functions without the particle. For example, consider two particles $P$ and $S$, where $S$ is a sphere centered at $\mathbf{r}_{S}$ with a radius $R$ large on a microscopic scale but much smaller than $\mathcal{R}_{g}$ (i.e., $R$ much smaller than $1 / \sqrt{t}$ ) and the smallest distance between $\mathbf{r}_{S}$ and the surface of $P$. We also assume that the distances of $\mathbf{r}_{1}$ and $\mathbf{r}_{2}$ from $\mathbf{r}_{S}$ are much larger than $R$. However, no restrictions are imposed on the length ratios $\left|\mathbf{r}_{1,2}-\mathbf{r}_{S}\right| / \mathcal{R}_{g}$ or on the ratio of $\mathcal{R}_{g}$ and the smallest distance between $\mathbf{r}_{S}$ and the surface of $P$. Similar to operator-product expansions ${ }^{19}$ the perturbation $S$ of small spatial extent can be represented by a series of pointoperators and the expansion reads

$$
\left\langle\varphi_{1,2}\right\rangle_{P, S}=\left\langle\varphi_{1,2} \cdot\left[1-\sum_{j} w_{j}\right]\right\rangle_{P},
$$

with

$$
w_{j}=A_{j} R^{d_{j}} O_{j}\left(\mathbf{r}_{S}\right) .
$$

Here the $O_{j}$ are the local operators

$$
\begin{aligned}
& \left(O_{\mathrm{I}}, O_{\mathrm{II}}, O_{\mathrm{III}}, O_{\mathrm{IV}}, O_{\mathrm{V}}, \ldots\right) \\
& \quad=\left(\frac{1}{2} \Phi^{2}, \frac{1}{4 !} \Phi^{4}, \frac{1}{2} t \Phi^{2}, \frac{1}{2}(\nabla \Phi)^{2}, \frac{1}{6 !} \Phi^{6}, \ldots\right),
\end{aligned}
$$

which are even in $\Phi$ and spatially isotropic and the $d_{j}$ are the dimensions

$$
\begin{aligned}
& \left(d_{\mathrm{I}}, d_{\mathrm{II}}, d_{\mathrm{III}}, d_{\mathrm{IV}}, d_{\mathrm{V}}, \ldots\right) \\
& \quad=(d-2,2(d-2), d, d, 3(d-2), \ldots)
\end{aligned}
$$

of the operators. The dimensionless amplitudes $A_{j}$ are given by

$$
\begin{aligned}
\left(A_{\mathrm{I}}, A_{\mathrm{II}}, A_{\mathrm{III}}, A_{\mathrm{IV}}, A_{\mathrm{V}}, \ldots\right) \\
=\left(\frac{4 \pi^{d / 2}}{\Gamma(\alpha)},-2 A_{\mathrm{I}}^{2}, \frac{\pi^{d / 2}}{\Gamma(\alpha)}\left(\frac{1}{\alpha+1}+\frac{1}{\alpha-1}\right),\right. \\
\\
\left.\quad \frac{2 \pi^{d / 2}}{\alpha \Gamma(\alpha)}, 8 A_{\mathrm{I}}^{3}, \ldots\right)
\end{aligned}
$$

as we show in Sec. II A below. $\Gamma$ is the Gamma function ${ }^{31}$ and

$$
\alpha=(d-2) / 2,
$$

i.e., the amplitudes only depend on the space dimension $d$. The brackets \langle\rangle$_{P, S}$ and \langle\rangle$_{P}$ on the left and right sides of Eq. (2.10) denote averages with two particles $P$ and $S$ present and with only one particle $P$ present, respectively. The quantities $\left\langle\varphi_{1,2} \cdot O_{j}\right\rangle_{P}$ on the right-hand side denote Wickdecompositions into factors $\langle\Phi \Phi\rangle$, which correspond to connected diagrams. For example,

$$
\begin{aligned}
\left\langle\varphi_{1,2} \cdot O_{\mathrm{V}}\right\rangle_{P}= & \frac{1}{8}\left\langle\Phi\left(\mathbf{r}_{1}\right) \Phi\left(\mathbf{r}_{S}\right)\right\rangle_{P}\left\langle\Phi\left(\mathbf{r}_{2}\right) \Phi\left(\mathbf{r}_{S}\right)\right\rangle_{P} \\
& \times\left(\left\langle\Phi\left(\mathbf{r}_{S}\right) \Phi\left(\mathbf{r}_{S}\right)\right\rangle_{P}\right)^{2} .
\end{aligned}
$$

Finally it is understood that ultraviolet-sensitive quantities such as the "closed loops" $\left\langle\Phi\left(\mathbf{r}_{S}\right) \Phi\left(\mathbf{r}_{S}\right)\right\rangle_{P}$ are to be evaluated in dimensional regularization. ${ }^{32}$

The partition function $Z_{P, S}\left(L ; \mathbf{r}_{1}, \mathbf{r}_{2}\right)$ for a chain with the two ends fixed and with two particles $P, S$ present follows from (2.10) by inverting the Laplace transform in Eq. (2.5). Volume-integrals of the partition function are also of interest. For example, the bulk-normalized density profile of chain ends in the polymer solution $\mathcal{E}_{P, S}$ in the presence of two particles is given by

$$
\mathcal{E}_{P, S}\left(L ; \mathbf{r}_{1}\right)=\int d \mathbf{r}_{2} Z_{P, S}\left(L ; \mathbf{r}_{1}, \mathbf{r}_{2}\right),
$$

where the $\mathbf{r}_{2}$-integration extends over all space except the volume occupied by the two particles. Equivalently one may define $Z_{P, S}$ to vanish for $\mathbf{r}_{1}$ or $\mathbf{r}_{2}$ inside $P$ or inside $S$ and integrate over all space. Of particular interest is the free energy $\left(\delta_{S} F\right)_{P}$ it costs to immerse the sphere $S$ in the polymer solution in the presence of particle $P$. This is given by ${ }^{17}$

$$
\left(\delta_{S} F\right)_{P}=p_{0} \int d \mathbf{r}_{1} \int d \mathbf{r}_{2}\left[Z_{P}\left(L ; \mathbf{r}_{1}, \mathbf{r}_{2}\right)-Z_{P, S}\left(L ; \mathbf{r}_{1}, \mathbf{r}_{2}\right)\right],
$$

where $p_{0}=n k_{B} T$ is the ideal gas pressure of the ideal polymer solution with chain density $n$. Here the $\mathbf{r}_{1}$ and $\mathbf{r}_{2}$-integrations extend over all space, $Z_{P}$ vanishes inside $P$, and $Z_{P, S}$ vanishes inside $P$ and $S$.

For a small sphere $S$ the volume-integrals appearing in the end-density (2.17) and the free energy (2.18) can be evaluated by means of slightly modified forms ${ }^{19}$ of the expansion (2.10). These read 


$$
\begin{aligned}
\int d \mathbf{r}_{2}\left\langle\varphi_{1,2}\right\rangle_{P, S}= & \int d \mathbf{r}_{2}\left\{\left\langle\varphi_{1,2} \cdot\left[1-\sum_{j} w_{j}\right]\right\rangle_{P}\right. \\
& \left.+D_{P}\left(\mathbf{r}_{1}, \mathbf{r}_{2}\right)\right\},
\end{aligned}
$$

for $\left|\mathbf{r}_{1}-\mathbf{r}_{S}\right| \gg R$ and

$$
\begin{aligned}
\int d \mathbf{r}_{1} \int d \mathbf{r}_{2}\left[\left\langle\varphi_{1,2}\right\rangle_{P, S}-\left\langle\varphi_{1,2}\right\rangle_{P}\right] \\
=\int d \mathbf{r}_{1} \int d \mathbf{r}_{2}\left\{\left\langle\varphi_{1,2} \cdot(-) \sum_{j} w_{j}\right\rangle_{P}+D_{P}\left(\mathbf{r}_{1}, \mathbf{r}_{2}\right)\right\} .
\end{aligned}
$$

Here the integrations on the right hand side extend over all space except the volume occupied by the particle $P$, and $D$ is the contact-term

$$
D_{P}\left(\mathbf{r}_{1}, \mathbf{r}_{2}\right)=\left\langle\varphi_{1,2}\right\rangle_{P} a R^{d}\left[\delta\left(\mathbf{r}_{1}-\mathbf{r}_{S}\right)+\delta\left(\mathbf{r}_{2}-\mathbf{r}_{S}\right)\right],
$$

with

$$
a=\frac{\pi^{d / 2}}{\Gamma(\alpha)} \frac{1}{\alpha+1},
$$

which only depends on the spatial dimension $d$. In the language of magnetism the left-hand side (lhs) of Eq. (2.19) is the local susceptibility $\chi_{P, S}\left(t ; \mathbf{r}_{1}\right)$ in the presence of $P$ and $S$, and the lhs of (2.20) is the change $\left(\delta_{S} \Xi(t)\right)_{P}$ in total susceptibility on introducing the sphere $S$ in the presence of $P$.

For the sake of generality and in order to disentangle ${ }^{33}$ the contributions of the various operators, we have presented the expansion for arbitrary spatial dimension $d>2$. Our main interest is in $d=3$ dimensions, and for later reference we note the corresponding dimensions

$$
\left(d_{\mathrm{I}}, d_{\mathrm{II}}, d_{\mathrm{III}}, d_{\mathrm{IV}}, d_{\mathrm{V}}\right)=(1,2,3,3,3)
$$

and amplitudes

$$
\begin{aligned}
\left(A_{\mathrm{I}}, A_{\mathrm{II}}, A_{\mathrm{III}}, A_{\mathrm{IV}}, A_{\mathrm{V}}\right)= & \left(4 \pi,-32 \pi^{2},\right. \\
& \left.-4 \pi / 3,4 \pi, 512 \pi^{3}\right)
\end{aligned}
$$

of the operators (2.12), as well as the corresponding contact amplitude $a=2 \pi / 3$. Thus for nonadsorbing ideal polymers in $d=3$, the depletion properties of a small spherical particle, such as the end density profile or the free energy it costs to immerse the particle, can be expanded in integer powers of the radius $R$, with the leading order contribution $\propto R$ determined by the operator $O_{\mathrm{I}}$, the next-to-leading order $\propto R^{2}$ by $O_{\mathrm{II}}$, and the next-to-next-to leading order $\propto R^{3}$ by the three operators $O_{\mathrm{III}}, O_{\mathrm{IV}}, O_{\mathrm{V}}$ and the contact term.

\section{A. Single spherical particle in a polymer solution}

In this simplest case the partition function $Z_{S}$, the end density $\mathcal{E}_{S}$, and the free energy cost $\left(\delta_{S} F\right)_{\text {bulk }}$ have been calculated for arbitrary size ratio $R / \mathcal{R}_{g}$ in closed analytic form, see, e.g., Ref. 15. As we shall see, these results confirm the small sphere expansions (2.10), (2.19), and (2.20) and allow one to obtain the results for the amplitudes $A_{j}$ and $a$ given in Eqs. (2.14) and (2.22) above. The single-sphere case is recovered from the system of two particles $P, S$ by moving particle $P$ to infinity. For the averages in Eqs. (2.10), (2.19), and (2.20), this implies

$$
\langle\rangle_{P, S} \rightarrow\langle\rangle_{S}, \quad\langle\rangle_{P} \rightarrow\langle\rangle_{\text {bulk }}
$$

while the amplitudes $A_{j}$ and $a$ remain unchanged. Here the subscript $S$ denotes a system with a single embedded spherical particle and the subscript "bulk" a system in an unbounded volume without a particle. We choose a coordinate system with origin at the center of the sphere.

We start with a discussion of Eq. (2.10). Its left-hand side is given, for arbitrary length ratios $R / r_{1,2}$ and $R \sqrt{t}$, by $^{15,16}$

$$
\left\langle\varphi_{1,2}\right\rangle_{S}=\left\langle\varphi_{1,2}\right\rangle_{\text {bulk }}+\delta_{S} G\left(t ; \mathbf{r}_{1}, \mathbf{r}_{2}\right),
$$

with

$$
\begin{aligned}
\delta_{S} G\left(t ; \mathbf{r}_{1}, \mathbf{r}_{2}\right)= & -\sum_{n=0}^{\infty} W_{n}^{(\alpha)}(\theta) \frac{1}{\left(r_{1} r_{2}\right)^{\alpha}} \frac{I_{\alpha+n}(R \sqrt{t})}{K_{\alpha+n}(R \sqrt{t})} \\
& \times K_{\alpha+n}\left(r_{1} \sqrt{t}\right) K_{\alpha+n}\left(r_{2} \sqrt{t}\right),
\end{aligned}
$$

where

$$
W_{n}^{(\alpha)}(\theta)=\frac{1}{2 \pi^{d / 2}} \Gamma(\alpha)(n+\alpha) C_{n}^{(\alpha)}(\cos \theta)
$$

depends on the angle $\theta$ between $\mathbf{r}_{1}$ and $\mathbf{r}_{2}$. Here $I, K$ are modified Bessel functions, and $C_{n}$ ultraspherical Gegenbauer polynomials. $^{31}$

On expanding the $\theta$-independent term $n=0$ in Eq. (2.27) for small $R \sqrt{t}$, one finds contributions of increasing order $R^{d_{\mathrm{I}}}, R^{d_{\mathrm{II}}}, R^{d_{\mathrm{III}}}, R^{d_{\mathrm{V}}}$, which can be identified with the terms $j=\mathrm{I}$,II,III,V on the right-hand side (rhs) of (2.10), provided the amplitudes $A_{j}$ are chosen as in Eq. (2.14). Here one uses that

$$
\langle\Phi(0) \Phi(\mathbf{r})\rangle_{\text {bulk }}=\frac{1}{(2 \pi)^{d / 2}}\left(\frac{\sqrt{t}}{r}\right)^{\alpha} K_{\alpha}(r \sqrt{t}),
$$

and

$$
\langle\Phi(0) \Phi(0)\rangle_{\mathrm{bulk}}=t^{\alpha} \frac{\Gamma(-\alpha)}{2^{d} \pi^{d / 2}}
$$

in dimensional regularization. The term $n=1$ in Eq. (2.27) contributes, in leading order, an expression proportional to $\cos \theta \cdot R^{d_{\mathrm{IV}}}$, which can be identified with the term $j=\mathrm{IV}$ on the rhs of (2.10), provided $A_{\mathrm{IV}}$ is chosen as in (2.14).

Next we consider the end density profile in Eq. (2.17) and its Laplace transform $\chi_{S}\left(t ; \mathbf{r}_{1}\right)$, given by the lhs of Eq. (2.19) with the replacements (2.25). For arbitrary ${ }^{15}$ length ratios $R / r_{1}, R \sqrt{t}$,

$$
\int d \mathbf{r}_{2}\left\langle\varphi_{1,2}\right\rangle_{S} \equiv \chi_{S}=\frac{1}{t}\left[1-\left(\frac{R}{r_{1}}\right)^{\alpha} \frac{K_{\alpha}\left(r_{1} \sqrt{t}\right)}{K_{\alpha}(R \sqrt{t})}\right] .
$$

The $R$-independent leading contribution $1 / t$ on the rhs equals the first term $\int d \mathbf{r}_{2}\left\langle\varphi_{1,2}\right\rangle_{\text {bulk }}$ in the small $R$ expansion on the rhs of Eq. (2.19). Expanding the second term on the rhs of

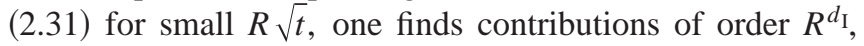
$R^{d_{\mathrm{II}}}$, and $R^{d_{\mathrm{V}}}$, which can be identified with the terms $j$ $=\mathrm{I}, \mathrm{II}, \mathrm{V}$ on the rhs of (2.19). There is also a contribution of 
order $R^{d}$ on the rhs of (2.31). This has the structure of the $j=$ III term on the rhs of (2.19) but a different amplitude. Here the contact-term comes to our aid, which has the same structure and ensures the validity of Eq. (2.19) in order $R^{d}$, provided the amplitude $a$ is chosen as in (2.22). Note that the derivative operator $O_{\mathrm{IV}}$ in (2.12) does not contribute to Eq. (2.19) in the single sphere case, since the quantity $\int d \mathbf{r}_{2}\left\langle\Phi\left(\mathbf{r}_{S}\right) \Phi\left(\mathbf{r}_{2}\right)\right\rangle_{\text {bulk }}$ is independent of $\mathbf{r}_{S}$.

Finally consider the free energy cost and the corresponding Laplace transform $\delta_{S} \Xi$ on the lhs of Eq. (2.20) with the replacements (2.25). For ${ }^{15}$ arbitrary $R \sqrt{t}$,

$$
\begin{aligned}
& \int d \mathbf{r}_{1} \int d \mathbf{r}_{2}\left[\left\langle\varphi_{1,2}\right\rangle_{S}-\left\langle\varphi_{1,2}\right\rangle_{\text {bulk }}\right] \\
& \equiv \delta_{S} \Xi=-R^{d} \frac{\Omega_{d}}{d} \frac{1}{t}-R^{d-1} \Omega_{d} \frac{K_{\alpha+1}(R \sqrt{t})}{t^{3 / 2} K_{\alpha}(R \sqrt{t})},
\end{aligned}
$$

where

$$
\Omega=\frac{2 \pi^{d / 2}}{\Gamma(d / 2)}
$$

is the surface of the $d$-dimensional sphere with radius unity. The first term on the rhs of Eq. (2.32) is proportional to the volume of the spherical particle and dominates if the particle is large. The second term arises from the depletion layer around the particle. It dominates in the small particle case and, apart from contributions of order $R^{d_{\mathrm{I}}}, R^{d_{\mathrm{II}}}$, and $R^{d_{\mathrm{v}}}$, which are identical to the $j=\mathrm{I}, \mathrm{II}, \mathrm{V}$ terms in the small radius expansion on the rhs of (2.20), also contains a contribution of order $R^{d}$. The sum of the two $R^{d}$-contributions in (2.32) can be identified with the sum of the $j=$ III term and the contact term on the rhs of Eq. (2.20). Again the derivative operator $O_{\mathrm{IV}}$ does not contribute to the rhs of (2.20).

We give more details for the case $d=3$, in which the expansion proceeds in integer powers of $R$. Then ${ }^{34}$ the end density profile $\mathcal{E}_{S}\left(L ; r_{1}\right)$ equals $1-\left(R / r_{1}\right) \operatorname{erfc}\left(\left(r_{1}-R\right) /\right.$ $\left(2 \mathcal{R}_{g}\right)$ ), and its Laplace transform, the local susceptibility on the right hand side of (2.31), is given by

$$
\begin{aligned}
\chi_{S} & =\frac{1}{t}\left[1-\frac{R}{r_{1}} e^{-\left(r_{1}-R\right) \sqrt{t}}\right] \\
& =\frac{1}{t}\left[1-\frac{1}{r_{1}} e^{-r_{1} \sqrt{t}}\left(R+R^{2} \sqrt{t}+\frac{1}{2} R^{3} t+\ldots\right)\right] .
\end{aligned}
$$

In the change of total susceptibility

$$
\delta_{S} \Xi=-\frac{4 \pi}{t^{2}}\left(R+R^{2} \sqrt{t}+\frac{1}{3} R^{3} t\right),
$$

which, apart from the sign, is the Laplace transform of the free energy cost $\delta_{S} F / p_{0}$ equal ${ }^{15,35}$ to $4 \pi \mathcal{R}_{g}^{2}(R$ $\left.+(2 / \sqrt{\pi}) R^{2} / \mathcal{R}_{g}+(1 / 3) R^{3} / \mathcal{R}_{g}^{2}\right)$, the terms of order $R^{3(d-2)}$ and $R^{d}$ from Eq. (2.32) cancel, and there are no contributions higher than $R^{3}$. The $R^{3}$-contributions on the right hand sides of Eqs. (2.34) and (2.35) arise from the linear combinations of terms due to $O_{\mathrm{III}}, O_{\mathrm{V}}$, and the contact term in the operator expansions on the right-hand sides of Eqs. (2.19) and (2.20) as follows: The factor $1 / 2$ in front of $R^{3}$ in Eq. (2.34) is reproduced in the form

$$
\frac{1}{4 \pi}\left(A_{\mathrm{III}}+\frac{A_{\mathrm{V}}}{128 \pi^{2}}-a\right)=\frac{1}{2},
$$

and the factor $1 / 3$ in front of $R^{3}$ in Eq. (2.35) in the form

$$
\frac{1}{4 \pi}\left(A_{\mathrm{III}}+\frac{A_{\mathrm{V}}}{128 \pi^{2}}-2 a\right)=\frac{1}{3} .
$$

This discussion of single sphere properties has familiarized us with the expansion and yielded the amplitudes $A_{j}$ and $a$. We now use the expansion to predict new analytic results for the interaction between a sphere and a wall and between two spheres.

\section{B. Interaction between a sphere and a wall}

Here we evaluate the free energy $\left(\delta_{S} F\right)_{W}$ it costs to immerse a small sphere with its center at a distance $z_{S}$ from a planar wall which is much larger than the sphere radius. We concentrate on $d=3$ dimensions, where

$$
\left(\delta_{S} F\right)_{W} / p_{0}=R g_{1}+R^{2} g_{2}+R^{3} g_{3}+\cdots .
$$

The term of order $R$ is known, ${ }^{17}$ and we calculate the contributions of order $R^{2}$ and $R^{3}$.

The expansion of the free energy follows from

$$
\left(\delta_{S} F\right)_{W} / p_{0}=-\mathcal{L} \int d \mathbf{r}_{1} \int d \mathbf{r}_{2}\left[\left\langle\varphi_{1,2}\right\rangle_{h \backslash S}-\left\langle\varphi_{1,2}\right\rangle_{h}\right]
$$

on substituting Eq. (2.20) on the right-hand side. To obtain Eq. (2.39) we have used Eqs. (2.18) and (2.5), (2.8). Here $\mathcal{L}$ denotes the inverse of the Laplace transform in (2.5). The planar wall at $z=0$ can be viewed as the surface of a huge particle $P$ which occupies the entire half space $z<0$. The volume available for the polymers is the entire half space $h$ with $z>0$, if the sphere is absent, and the half space minus the volume occupied by the sphere, $h \backslash S$, if the sphere is present. We have changed the notation

$$
\langle\rangle_{P, S} \rightarrow\langle\rangle_{h \backslash S}, \quad\langle\rangle_{P} \rightarrow\langle\rangle_{h},
$$

correspondingly.

Substituting this version of the small sphere expansion (2.20) on the rhs of (2.39) and applying Wick's theorem leads to

$$
\begin{aligned}
& g_{1}=A_{\mathrm{I}} \mathcal{L}\left[\chi_{h}\left(z_{S}\right)\right]^{2}, \\
& g_{2}=\left(A_{\text {II }} / 2\right) \mathcal{L} e_{h}\left(z_{S}\right)\left[\chi_{h}\left(z_{S}\right)\right]^{2},
\end{aligned}
$$

and

$$
g_{3}=g_{3, \mathrm{III}}+g_{3, \mathrm{IV}}+g_{3, \mathrm{~V}}+g_{3, D},
$$

with

$$
\begin{aligned}
& g_{3, \mathrm{III}}=A_{\mathrm{III}} \mathcal{L} t\left[\chi_{h}\left(z_{S}\right)\right]^{2}, \\
& g_{3, \mathrm{IV}}=A_{\mathrm{IV}} \mathcal{L}\left[\partial_{z_{S}} \chi_{h}\left(z_{S}\right)\right]^{2}, \\
& g_{3, \mathrm{~V}}=\left(A_{\mathrm{V}} / 8\right) \mathcal{L}\left[e_{h}\left(z_{S}\right) \chi_{h}\left(z_{S}\right)\right]^{2}, \\
& g_{3, D}=-2 a \mathcal{L} \chi_{h}\left(z_{S}\right) .
\end{aligned}
$$

Here 


$$
\chi_{h}\left(z_{S}\right) \equiv \int d \mathbf{r}_{1}\left\langle\Phi\left(\mathbf{r}_{1}\right) \Phi\left(\mathbf{r}_{S}\right)\right\rangle_{h}=\frac{1}{t}\left(1-e^{-z_{S} \sqrt{t}}\right),
$$

where the $\mathbf{r}_{1}$-integration extends over the half space $h$, is the local susceptibility in the half space, and

$$
e_{h}\left(z_{S}\right) \equiv\left\langle\Phi\left(\mathbf{r}_{S}\right) \Phi\left(\mathbf{r}_{S}\right)\right\rangle_{h}=-\frac{\sqrt{t}}{4 \pi}\left(1+\frac{1}{2 z_{S} \sqrt{t}} e^{-2 z_{S} \sqrt{t}}\right)
$$

is the contribution from the closed loop in dimensional regularization. For $z_{S} \rightarrow \infty$ the quantities $\chi_{h}$ and $e_{h}$ approach their bulk expressions $1 / t$ and $-\sqrt{t} /(4 \pi)$, see Eq. (2.30). Inserting the expressions (2.48) and (2.49) for $\chi_{h}$ and $e_{h}$ into Eqs. (2.41), (2.42), and (2.44)-(2.47) and performing the inverse Laplace transforms leads to the known result

$$
g_{1}=4 \pi \mathcal{R}_{g}^{2} \mathcal{M}_{h},
$$

where

$$
\mathcal{M}_{h}=1+4\left[-2 \epsilon_{2}(y / 2)+\epsilon_{2}(y)\right]
$$

is the bulk normalized monomer density profile in the half space, ${ }^{27}$ and to the new results

$$
\begin{aligned}
g_{2}= & 8 \pi \mathcal{R}_{g}\left\{\frac{1}{\sqrt{\pi}}-2 \epsilon_{1}(y / 2)+\epsilon_{1}(y)\right. \\
& \left.+\frac{1}{y}\left[\epsilon_{2}(y)-2 \epsilon_{2}(3 y / 2)+\epsilon_{2}(2 y)\right]\right\}
\end{aligned}
$$

and

$$
\begin{aligned}
g_{3}= & 2 \pi\left\{\frac{2}{3}-2 \epsilon_{0}(y / 2)+\frac{10}{3} \epsilon_{0}(y)\right. \\
& +\frac{4}{y}\left[\epsilon_{1}(y)-2 \epsilon_{1}(3 y / 2)+\epsilon_{1}(2 y)\right] \\
& \left.+\frac{2}{y^{2}}\left[\epsilon_{2}(2 y)-2 \epsilon_{2}(5 y / 2)+\epsilon_{2}(3 y)\right]\right\} .
\end{aligned}
$$

Here we have introduced the scaled distance

$$
y=z_{S} / \mathcal{R}_{g}
$$

of the sphere center from the wall and the notation

$$
\epsilon_{n}(x)=\mathrm{i}^{n} \operatorname{erfc}(x)
$$

for the $\mathrm{n}$-fold iterated complementary error function. ${ }^{31}$ For small $y$

$$
g_{3} \rightarrow 2 \pi\{5 / 2-31 y /(6 \sqrt{\pi})+\ldots\} .
$$

Our results imply the finite limit ${ }^{36}$

$$
\left(\delta_{S} F\right)_{W} / p_{0} \rightarrow \pi R z_{S}^{2}\left[4+2 R / z_{S}+5\left(R / z_{S}\right)^{2}+\cdots\right]
$$

of the immersion free energy for $\mathcal{R}_{g} \rightarrow \infty$. The expansion on the right-hand side of (2.57) applies for small $R / z_{S}$ and is in agreement with the finite limit expression for arbitrary $R / z_{S}$ given in Eqs. (2.3), (2.14a), and (2.14b) of Ref. 17 where $\left(\delta_{S} F\right)_{W}, p_{0}$, and $z_{S}$ have been denoted by $F, p$, and $D$ $+R$, respectively.

In Figs. 2 and 3 we compare the analytic expressions

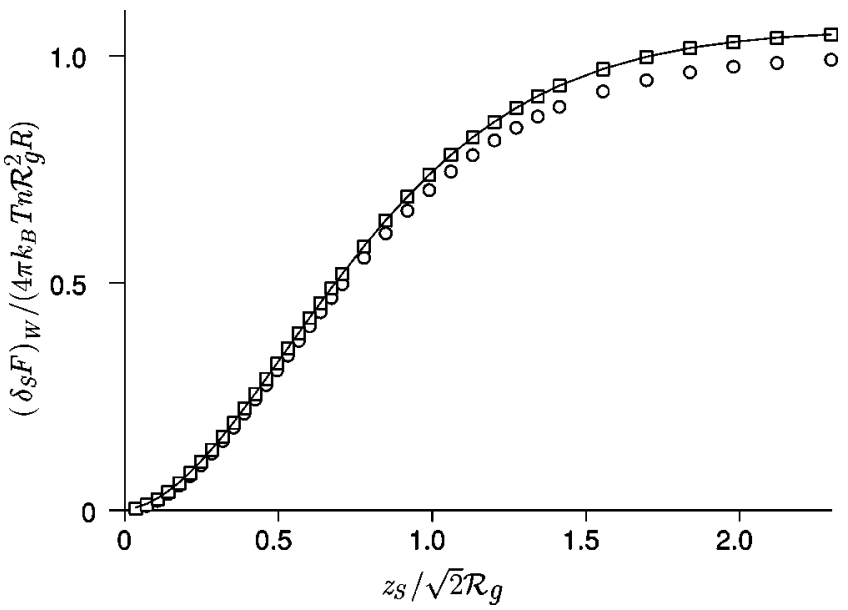

FIG. 2. Free energy cost $\left(\delta_{S} F\right)_{W}$ of immersing a small spherical particle at a distance $z_{S}$ from a planar wall. In the case shown, with the particle radius smaller by a factor twenty than the polymer radius of gyration, the sum (squares) of the leading order contribution proportional to $R$ (circles) and the next to leading order contribution proportional to $R^{2}$ which follow from Eqs. (2.38) and (2.50) -(2.52) is nearly indistinguishable from the complete result (full line), obtained numerically by the multiple scattering method of Ref. 17.

(2.52) and (2.53) for the free energy cost (2.38) in next-toand next-to-next-to-leading order in the particle size with a numerical calculation using a multiple scattering formalism ${ }^{17}$ in order to solve the diffusion equation. The agreement is excellent and indicates that the proposed operator expansion is a reliable and useful tool to describe the small particle behavior even beyond the leading order. Note the interesting nonmonotonic dependence of $g_{3}$ on $z_{S} / \mathcal{R}_{g}$. In the next chapter we apply the operator expansion approach to the case

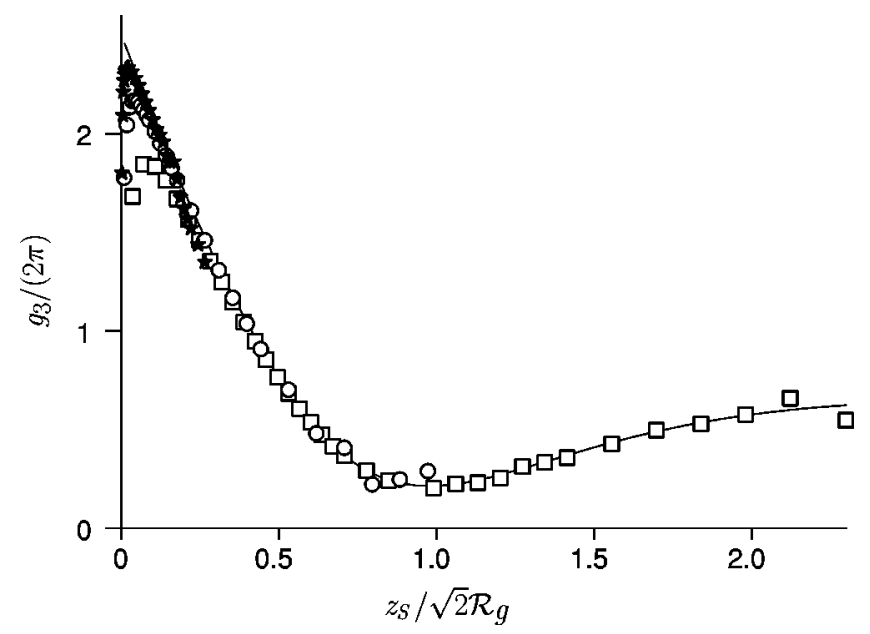

FIG. 3. Next-to-next-to-leading contribution of the free energy cost $\left(\delta_{S} F\right)_{W}$ of immersing a small spherical particle near a planar wall, which is proportional to $R^{3}$ and characterized by the function $g_{3}$ in Eq. (2.38). The analytic prediction (2.53) of the operator expansion (full line) is compared with a direct numerical evaluation of the free energy expression (2.39) following the method of Ref. 17 for size ratios $R / \mathcal{R}_{g}=0.05$ (squares), 0.0125 (circles), and 0.0031 (asterisks). For $R \ll z_{S}, \mathcal{R}_{g}$ the data convincingly collapse onto the predicted curve. Note that the ranges of numerical data shown shrink proportional to $R$. This is due to subtraction errors which begin at a constant ratio $R / z_{S}$. 
of small anisotropic particles, where numerical results are not available.

\section{Interaction between two spheres}

In this section we consider the free energy of interaction $F_{\text {int }}$ of two small spheres $S$ and $S^{\prime}$ with radii $R$ and $R^{\prime}$, which is induced by ideal, free, nonadsorbing polymer chains and evaluate the next correction to the known ${ }^{16}$ leading-order result. These are the contributions

$$
\begin{aligned}
\sigma_{1} & =-\mathcal{L} \int d \mathbf{r}_{1} \int d \mathbf{r}_{2}\left\langle\varphi_{1,2} \cdot w_{\mathrm{I}} \cdot w_{\mathrm{I}}^{\prime}\right\rangle_{\text {bulk }} \\
& =-\left(R R^{\prime}\right)^{2 \alpha} A_{\mathrm{I}}^{2} 2 \mathcal{L} \frac{1}{t^{2}} G_{\mathrm{b}}(r)
\end{aligned}
$$

and

$$
\begin{aligned}
\sigma_{\mathrm{nl}}= & -\mathcal{L} \int d \mathbf{r}_{1} \int d \mathbf{r}_{2}\left\langle\varphi_{1,2} \cdot\left[w_{\mathrm{I}} \cdot w_{\mathrm{II}}^{\prime}+w_{\mathrm{II}} \cdot w_{\mathrm{I}}^{\prime}\right]\right\rangle_{\mathrm{bulk}} \\
= & -\left(R R^{\prime}\right)^{2 \alpha}\left(R^{2 \alpha}+\left(R^{\prime}\right)^{2 \alpha}\right) A_{\mathrm{I}} A_{\mathrm{II}} \mathcal{L} \frac{1}{t^{2}} \\
& \times\left[G_{\mathrm{b}}(r)\langle\Phi(0) \Phi(0)\rangle_{\mathrm{bulk}}+\frac{1}{2} G_{\mathrm{b}}^{2}(r)\right]
\end{aligned}
$$

to

$$
F_{\text {int }} / p_{0}=\sigma_{1}+\sigma_{\mathrm{nl}}+\cdots .
$$

Here $w_{j}^{\prime}$ follows from $w_{j}$ in Eq. (2.11) on replacing the radius $R$ by $R^{\prime}$ and the sphere center $\mathbf{r}_{S}$ by $\mathbf{r}_{S^{\prime}}$, where

$$
r=\left|\mathbf{r}_{S}-\mathbf{r}_{S^{\prime}}\right|
$$

is the distance between the sphere centers which is much larger than $R$ and $R^{\prime}$ and

$$
G_{\mathrm{b}}(r)=\left\langle\Phi\left(\mathbf{r}_{S}\right) \Phi\left(\mathbf{r}_{S^{\prime}}\right)\right\rangle_{\text {bulk }}
$$

is the order parameter correlation function in unbounded bulk. Equations (2.58) and (2.59) can be derived from Eqs. (2.18) and (2.20) by replacing the particle $P$ by the second sphere $S^{\prime}$, using Wick's theorem, and expanding the $S^{\prime}$-averages $\left\langle\Phi\left(\mathbf{r}_{1,2}\right) \Phi\left(\mathbf{r}_{S}\right)\right\rangle_{S^{\prime}}$ and $\left\langle\Phi\left(\mathbf{r}_{S}\right) \Phi\left(\mathbf{r}_{S}\right)\right\rangle_{S^{\prime}}$ for small $R^{\prime}$ in a small sphere expansion for $S^{\prime}$.

In the case of $d=3$ dimensions, where

$$
G_{\mathrm{b}}(r)=\frac{1}{4 \pi r} e^{-r \sqrt{t}}
$$

one obtains

$$
\sigma_{1}=-R R^{\prime} \mathcal{R}_{g}^{2} \frac{32 \pi}{r} \epsilon_{2}\left(\frac{r}{2 \mathcal{R}_{g}}\right)
$$

for the leading contribution, known from Eqs. (1.16a), (3.9), and (3.10) in Ref. 16, and for the next-to-leading contribution the new result

$\sigma_{\mathrm{nl}}=R R^{\prime}\left(R+R^{\prime}\right) 16 \pi\left[-\frac{\mathcal{R}_{g}}{r} \epsilon_{1}\left(\frac{r}{2 \mathcal{R}_{g}}\right)+\frac{\mathcal{R}_{g}^{2}}{r^{2}} \epsilon_{2}\left(\frac{r}{\mathcal{R}_{g}}\right)\right]$,

with the error functions $\epsilon_{n}$ defined in Eq. (2.55) above. We give the limiting behavior

$$
\sigma_{1}+\sigma_{\mathrm{nl}} \rightarrow-8 \pi R R^{\prime} \frac{\mathcal{R}_{g}^{2}}{r}\left[1-\frac{1}{2} \frac{R+R^{\prime}}{r}\right]
$$

for $r \ll \mathcal{R}_{g}$ and

$\sigma_{1}+\sigma_{\mathrm{nl}} \rightarrow-64 \sqrt{\pi} R R^{\prime} \frac{\mathcal{R}_{g}^{5}}{r^{4}}\left[1+\frac{1}{4} \frac{\left(R+R^{\prime}\right) r}{\mathcal{R}_{g}^{2}}\right] e^{-r^{2} /\left(4 \mathcal{R}_{g}^{2}\right)}$

for $r \gg \mathcal{R}_{g}$. Note that $\sigma_{\mathrm{nl}}$, which is of third order in the particle radii, changes sign on increasing $r / \mathcal{R}_{g}$. Thus the next-to-leading contribution weakens the attraction between spheres for $r \ll \mathcal{R}_{g}$ and strengthens it for $r \gg \mathcal{R}_{g}$.

Equation (2.66) provides the first two terms in an expansion for $R, R^{\prime} \ll r$ of the finite limit of $F_{\text {int }} /\left(p_{0} \mathcal{R}_{g}^{2}\right)$ for $\mathcal{R}_{g}$ $\rightarrow \infty$. For $R=R^{\prime}$ the expansion is in agreement with the finite limit expression for arbitrary $R / r$ given in Eqs. (3.13), (3.15), (3.18a), and (3.18b) of Ref. 16 where $F_{\text {int }} / p_{0}$ and $r$ have been denoted by $f_{A, B}^{(2)}$ and $r_{A, B}$, respectively. $A_{D, D} R^{D-1 / \nu} \mathcal{R}_{x}^{1 / \nu}$ in Eq. (3.13) of Ref. 16 equals $4 \pi R \mathcal{R}_{g}^{2}$ for ideal polymers in $d=3$ dimensions.

\section{DEPLETION FOR A SMALL ANISOTROPIC PARTICLE}

Consider a particle with the shape of a prolate or oblate ellipsoid of revolution in $d=3$ dimensions, as shown in Fig. 1. We introduce the interfocal distance $2 f$, which is related to the long and short axes $l$ and $s$ of the ellipsoid by

$$
l^{2}-s^{2}=(2 f)^{2},
$$

and a dimensionless parameter

$$
\xi=(l, s) /(2 f) \text { for (prolate, oblate) }
$$

characterizing the degree of shape anisotropy of the particle. For $\xi \rightarrow \infty$ the prolate or oblate ellipsoid becomes a sphere of radius $l / 2=s / 2$, for $\xi \rightarrow 1$ the prolate ellipsoid becomes an infinitely thin needle of length $l=2 f$, and for $\xi \rightarrow 0$ the oblate ellipsoid becomes an infinitely thin circular disk of radius $l / 2=f$. The parameter $\xi$ also appears on introducing spheroidal coordinate systems. ${ }^{37}$

For a small ellipsoid with $l$ and $s$ much smaller than the other mesoscopic lengths, a small particle expansion again applies. However, anisotropic operators such as

$$
O_{\mathrm{VI}}=\frac{1}{2}\left(\partial_{\|} \Phi\right)^{2}, \quad O_{\mathrm{VII}}=\frac{1}{2} \Phi\left(\partial_{\|}^{2} \Phi\right),
$$

with dimensions

$$
d_{\mathrm{VI}}=d_{\mathrm{VII}}=3 \text {, }
$$

also contribute. Here

$$
\partial_{\|}=\sum_{\alpha=1}^{3} \rho_{\alpha} \partial_{\alpha}
$$

is a derivative along the axis of revolution of the ellipsoid characterized by the unit vector $\vec{\rho}$. Note that other uniaxially anisotropic operators such as $\partial_{\|}^{2} \Phi^{2}$ or $\Phi \Delta_{\perp} \Phi$ can be reduced to combinations of $O_{\mathrm{VI}}$ and $O_{\mathrm{VII}}$ or of $O_{\mathrm{VII}}$ and an isotropic operator. With $f$ as the length unit for the particle size, the weights $w_{j}$, which are the counterparts for the ellipsoid of the expressions (2.11) for the sphere, take the form 


$$
w_{j}=B_{j}(\xi) f^{d_{j}} O_{j}\left(\mathbf{r}_{E}\right),
$$

with amplitudes $B$ which are functions of the anisotropy parameter $\xi$.

The most important amplitudes are those of the leading isotropic operator $O_{\mathrm{I}}$ in (2.12), with $d_{\mathrm{I}}=1$ given in (2.23), and of the leading anisotropic operators $O_{\mathrm{VI}}$ and $O_{\mathrm{VII}}$ in (3.3), for which one finds

$$
B_{\mathrm{I}}=4 \pi\left(\frac{1}{Q_{0}(\xi)}, \frac{-i}{Q_{0}(i \xi)}\right)
$$

and

$$
\begin{aligned}
& B_{\mathrm{VI}}=\frac{4 \pi}{3}\left(\frac{P_{1}(\xi)}{Q_{1}(\xi)}+2 \frac{P_{1}^{1}(\xi)}{Q_{1}^{1}(\xi)}, i \frac{P_{1}(i \xi)}{Q_{1}(i \xi)}+2 i \frac{P_{1}^{1}(i \xi)}{Q_{1}^{1}(i \xi)}\right), \\
& B_{\mathrm{VII}}=\frac{1}{3}\left(B_{\mathrm{I}},-B_{\mathrm{I}}\right)
\end{aligned}
$$

in the (prolate, oblate) cases, respectively, as we show in Sec. III A below. Here $P$ and $Q$ are Legendre functions of the first and second kind, as defined in Chap. 8 of Ref. 31. We give the simple representations

$$
4 \pi / B_{\mathrm{I}}=\left(\frac{1}{2} \ln \frac{\xi+1}{\xi-1}, \arctan \frac{1}{\xi}\right)=\left(\operatorname{Arcosh} \frac{l}{s}, \arccos \frac{s}{l}\right)
$$

of the positive amplitudes $B_{\mathrm{I}}$.

We note important special cases of Eqs. (3.7)-(3.9). (i) In the limit $\xi \rightarrow \infty$ of a weakly deformed sphere of (prolate, oblate) shape, Eq. (3.7) yields

$$
B_{\mathrm{I}} \cdot f \rightarrow 4 \pi \xi(1,1) \cdot f \rightarrow 4 \pi R(1,1),
$$

where in the last step we have used that the radius $R$ of the sphere equals $\xi f$, see Eq. (3.2). Comparing Eq. (3.6) with (2.11) shows that this is consistent with the value $A_{\mathrm{I}}=4 \pi$ in Eq. (2.24) of the small sphere expansion in Sec. II. In the limit $\xi \rightarrow \infty$ Eqs. (3.8) and (3.9) give

$$
B_{\mathrm{VI}} \cdot f^{3} \rightarrow \frac{12 \pi}{5} \xi(1,-1) \cdot f^{3} \rightarrow \frac{12 \pi}{5} R^{2}(l-s)(1,-1)
$$

and

$$
B_{\mathrm{VII}} \cdot f^{3} \rightarrow \frac{4 \pi}{3} \xi(1,-1) \cdot f^{3} \rightarrow \frac{4 \pi}{3} R^{2}(l-s)(1,-1)
$$

for the amplitudes of the anisotropic operators, where Eqs. (3.1) and (3.2) have been used in the last steps. As expected, the couplings to the anisotropic operators have opposite signs for weakly deformed spheres of prolate and oblate shape and vanish in the spherical limit $l=s$.

(ii) For a thin needle, i.e., for $\xi \rightarrow 1$ in the prolate case, Eq. (3.7) leads to

$$
B_{\mathrm{I}} \cdot f \rightarrow \frac{4 \pi}{\frac{1}{2} \ln \frac{2}{\xi-1}} \cdot f \rightarrow \frac{2 \pi l}{\ln (2 l / s)},
$$

where Eqs. (3.1) and (3.2) have been used in the last step. The anisotropy amplitudes follow in the same way from Eqs. (3.8) and (3.9) and are given by

$$
B_{\mathrm{VI}} \cdot f^{3} \rightarrow \frac{4 \pi / 3}{\frac{1}{2} \ln \frac{2}{\xi-1}-1} \cdot f^{3} \rightarrow \frac{\pi}{6} \frac{l^{3}}{\ln (2 l / s)-1}
$$

and

$$
B_{\mathrm{VII}} \cdot f^{3} \rightarrow \frac{4 \pi / 3}{\frac{1}{2} \ln \frac{2}{\xi-1}} \cdot f^{3} \rightarrow \frac{\pi}{6} \frac{l^{3}}{\ln (2 l / s)} .
$$

Note that the $B_{j}$ vanish logarithmically as $s / l$ tends to zero. The reason is that an infinitely thin needle does not affect the polymer conformations in three spatial dimensions. This is different for the case of an infinitely thin disk, to which we now turn.

(iii) For a circular disk, i.e., for $\xi \rightarrow 0$ in the oblate case, Eq. (3.7) implies

$$
B_{\mathrm{I}} \cdot f \rightarrow 8 \cdot f \rightarrow 8 R_{\mathrm{disk}},
$$

and Eqs. (3.8) and (3.9) yield

$$
B_{\mathrm{VI}} \cdot f^{3} \rightarrow-\frac{16}{3} \cdot f^{3} \rightarrow-\frac{16}{3} R_{\text {disk }}^{3}
$$

and

$$
B_{\mathrm{VII}} \cdot f^{3} \rightarrow-\frac{8}{3} \cdot f^{3} \rightarrow-\frac{8}{3} R_{\text {disk }}^{3},
$$

where $R_{\text {disk }}$ is the radius of the disk.

\section{A. A single ellipsoid}

We first describe how the results (3.7) and (3.8), (3.9) for the amplitudes $B_{\mathrm{I}}$ and $B_{\mathrm{VI}}, B_{\mathrm{VII}}$ can be obtained from the order-parameter correlation function $\left\langle\varphi_{1,2}\right\rangle_{E}$ outside a single ellipsoid $E$. In this subsection it is convenient to use a coordinate system with the origin at the center and the $z$ axis along the axis of revolution of the ellipsoid. For the two points $\mathbf{r}_{1}, \mathbf{r}_{2}$ with Cartesian coordinates ${ }^{38}$

$$
\mathbf{r}_{k}=\left(r_{k, \perp} \cos \varphi_{k}, \quad r_{k, \perp} \sin \varphi_{k}, z_{k}\right) ; \quad k=1,2,
$$

we introduce spheroidal coordinates $\xi_{k}$ and $\eta_{k}$ which are related to the distances $r_{k, \perp}$ from the $z$ axis and the $z$ components $z_{k}$ as in Ref. 37. For the prolate case with the foci located on the $z$ axis at $z= \pm f$,

$$
\begin{aligned}
& 2 f \xi_{k}=r_{k,+}+r_{k,-}, \\
& 2 f \eta_{k}=r_{k,+}-r_{k,-},
\end{aligned}
$$

with

$$
r_{k, \pm}=\sqrt{r_{k, \perp}^{2}+\left(z_{k} \pm f\right)^{2}} .
$$

For the oblate case

$$
\begin{aligned}
& 2 f \sqrt{1+\xi_{k}^{2}}=r_{k,+}+r_{k,-}, \\
& 2 f \sqrt{1-\eta_{k}^{2}}=r_{k,+}-r_{k,-},
\end{aligned}
$$

with

$$
r_{k, \pm}=\sqrt{z_{k}^{2}+\left(r_{k, \perp} \pm f\right)^{2}} .
$$

The form of 


$$
\left\langle\varphi_{1,2}\right\rangle_{E}=\left\langle\varphi_{1,2}\right\rangle_{\text {bulk }}+\delta_{E} G,
$$

which satisfies the Ornstein-Zernicke equation (2.6) outside the ellipsoid, i.e., for $\xi_{1}>\xi, \xi_{2}>\xi$, and vanishes for $\mathbf{r}_{1}$ or $\mathbf{r}_{2}$ on its surface, i.e., for $\xi_{1}=\xi$ or $\xi_{2}=\xi$, can also be obtained with the help of Ref. 37. In order to determine $B_{\mathrm{I}}$ and $B_{\mathrm{VI}}$, $B_{\mathrm{VII}}$ it is sufficient to consider $\left\langle\varphi_{1,2}\right\rangle_{E}$ right at the critical point $t=0$, where

$$
\delta_{E} G=-\sum_{m, n} \gamma_{m, n},
$$

with

$$
\gamma_{m, n}=\mathcal{C}_{m, n} P_{n}^{m}\left(\eta_{1}\right) P_{n}^{m}\left(\eta_{2}\right) \cos \left(m\left(\varphi_{1}-\varphi_{2}\right)\right) \cdot \beta .
$$

Here

$$
\beta=\frac{P_{n}^{m}(\xi)}{Q_{n}^{m}(\xi)} Q_{n}^{m}\left(\xi_{1}\right) Q_{n}^{m}\left(\xi_{2}\right)
$$

in the prolate case, while

$$
\beta=i \frac{P_{n}^{m}(i \xi)}{Q_{n}^{m}(i \xi)} Q_{n}^{m}\left(i \xi_{1}\right) Q_{n}^{m}\left(i \xi_{2}\right)
$$

in the oblate case. The double sum in Eq. (3.28) is over all integer values $m, n$ obeying $0 \leqslant m \leqslant n, P$ and $Q$ are Legendre functions, ${ }^{31}$ and the coefficients $\mathcal{C}_{m, n}$ are given by

$$
\mathcal{C}_{m, n}=\frac{1}{f}(-)^{m}\left(\frac{(n-m) !}{(n+m) !}\right)^{2} \frac{2 n+1}{2 \pi}
$$

for $m=1,2,3, \ldots$ and

$$
\mathcal{C}_{0, n}=\frac{1}{f} \frac{2 n+1}{4 \pi}
$$

for $m=0$.

The amplitudes $B_{\mathrm{I}}$ and $B_{\mathrm{VI}}, B_{\mathrm{VII}}$ follow from $\delta_{E} G$ for small $f / r_{1}, f / r_{2}$, i.e., for small

$$
\frac{1}{\xi_{k}}=\frac{f}{r_{k}}\left[1 \mp \frac{f^{2}}{2 r_{k}^{2}}\left(1-\cos ^{2} \theta_{k}\right)+\ldots\right] .
$$

Here the upper and lower signs apply in the prolate and oblate cases,

$$
\cos \theta_{k}=z_{k} / r_{k}
$$

determines the azimuthal angles $\theta_{k}$, and Eqs. (3.21) and (3.24) have been used to derive (3.34). The two factors $Q_{n}^{m}\left(\xi_{k}\right)$ or $Q_{n}^{m}\left(i \xi_{k}\right)$ in (3.30) or (3.31) must be expanded for large arguments, ${ }^{31}$ and the orders $f$ and $f^{3}$ of $\delta_{E} G$ which we need are contained in the three contributions $\gamma_{0,0}, \gamma_{0,1}$, and $\gamma_{1,1}$. On using

$$
\eta_{k}=\cos \theta_{k}+\cdots,
$$

which follows from (3.22) and (3.25), the expansion yields

$$
\gamma_{0,0} \rightarrow B_{\mathrm{I}} f \tau_{\mathrm{I}}+B_{\mathrm{VII}} f^{3} \tau_{\mathrm{VII}},
$$

with $B_{\mathrm{I}}$ and $B_{\mathrm{VII}}$ from (3.7) and (3.9),

$$
\tau_{\mathrm{I}}=\frac{1}{4 \pi r_{1} 4 \pi r_{2}}=\left\langle\varphi_{1,2} \cdot O_{\mathrm{I}}\right\rangle_{\text {bulk }},
$$

and

$$
\begin{aligned}
\tau_{\mathrm{VII}} & =-\frac{1}{2} \frac{1}{4 \pi r_{1} 4 \pi r_{2}}\left\{\frac{1}{r_{1}^{2}}\left[1-3 \cos ^{2} \theta_{1}\right]+(1 \rightarrow 2)\right\} \\
& =\left\langle\varphi_{1,2} \cdot O_{\mathrm{VII}}\right\rangle_{\text {bulk }} .
\end{aligned}
$$

Moreover,

$$
\gamma_{0,1} \rightarrow\left(B_{\mathrm{IV}}+B_{\mathrm{VI}}\right) f^{3} \tau_{\mathrm{VI}}
$$

and

$$
\gamma_{1,1} \rightarrow B_{\mathrm{IV}} f^{3} \tau,
$$

with

$$
B_{\mathrm{IV}}=-\frac{8 \pi}{3}\left(\frac{P_{1}^{1}(\xi)}{Q_{1}^{1}(\xi)}, i \frac{P_{1}^{1}(i \xi)}{Q_{1}^{1}(i \xi)}\right)
$$

for the (prolate, oblate) case and $B_{\mathrm{VI}}$ given in Eq. (3.8), and with

$$
\tau_{\mathrm{VI}}=\frac{\cos \theta_{1} \cos \theta_{2}}{4 \pi r_{1}^{2} 4 \pi r_{2}^{2}}=\left\langle\varphi_{1,2} \cdot O_{\mathrm{VI}}\right\rangle_{\mathrm{bulk}}
$$

and

$$
\tau=\frac{\sin \theta_{1} \sin \theta_{2} \cos \left(\varphi_{1}-\varphi_{2}\right)}{4 \pi r_{1}^{2} 4 \pi r_{2}^{2}}=\left\langle\varphi_{1,2} \cdot\left(O_{\mathrm{IV}}-O_{\mathrm{VI}}\right\rangle_{\text {bulk }} .\right.
$$

$B_{\text {IV }}$ in (3.42) is indeed the amplitude in Eq. (3.6) corresponding to the derivative operator $O_{\mathrm{IV}}$ in (2.12), since the sum $\gamma_{0,1}+\gamma_{1,1}$ equals $f^{3}\left\langle\varphi_{1,2} \cdot\left(B_{\mathrm{IV}} O_{\mathrm{IV}}+B_{\mathrm{VI}} O_{\mathrm{VI}}\right)\right\rangle_{\text {bulk }}$.

For $t>0$ the expansion of $\left\langle\varphi_{1,2}\right\rangle_{E}$ also contains contributions from the operators $O_{\mathrm{II}}, O_{\mathrm{III}}$, and $O_{\mathrm{V}}$ in Eq. (2.12), and one finds, for example, that $B_{\mathrm{II}}=-2 B_{\mathrm{I}}^{2}$. For most of the quantities of interest considered below we only discuss the leading isotropic and anisotropic contributions, for which $B_{\mathrm{II}}, \ldots, B_{\mathrm{V}}$ and the counterpart $b(\xi) f^{3}$ for the ellipsoid of the quantity $a R^{d}$ for the sphere in the contact-term $D$ in (2.21) do not contribute.

Next we consider the bulk-normalized density $\mathcal{E}_{E}\left(L ; \mathbf{r}_{1}\right)$ of polymer ends around a single ellipsoidal particle. This is given by the inverse Laplace transform of Eq. (2.19), with the averages \langle\rangle$_{P, S}$ and \langle\rangle$_{P}$ replaced by \langle\rangle$_{E}$ and \langle\rangle$_{\text {bulk }}$ and the amplitudes $A_{j} R^{d_{j}}$ and $a R^{d}$ in $w_{j}$ and $D$ by $B_{j} f^{d_{j}}$ and $b f^{3}$. The leading contribution

$$
\begin{aligned}
& {\left[\mathcal{E}_{E}\left(L ; \mathbf{r}_{1}\right)-1\right]_{\text {leading isotropic }}=-\frac{B_{\mathrm{I}} f}{4 \pi r_{1}} \operatorname{erfc} \frac{r_{1}}{2 \mathcal{R}_{g}} ;} \\
& \quad l, s \ll r_{1}, \mathcal{R}_{g}
\end{aligned}
$$

is isotropic and determined by the operator $O_{\mathrm{I}}$, and the leading anisotropic contribution

$$
\left[\mathcal{E}_{E}\left(L ; \mathbf{r}_{1}\right)\right]_{\text {leading anisotropic }}=-\frac{B_{\mathrm{VII}} f^{3}}{8 \pi} \kappa ; \quad l, s \ll r_{1}, \mathcal{R}_{g}
$$

is determined only by $O_{\mathrm{VII}}$, since the contribution of $O_{\mathrm{VI}}$ to $\mathcal{E}_{E}$ vanishes. Here

$$
\kappa=\left[\partial_{z_{1}}^{2}\left(\frac{1}{r_{1}} \operatorname{erfc} \frac{r_{1}}{2 \mathcal{R}_{g}}\right)\right]_{\text {anisotropic }}=\lambda \cos ^{2} \theta_{1},
$$

with 


$$
\begin{aligned}
& \lambda=\frac{1}{\left(2 \mathcal{R}_{g}\right)^{3}}\left[\frac{3}{\varrho_{1}^{3}} \operatorname{erfc} \varrho_{1}+\frac{4}{\sqrt{\pi}} e^{-\varrho_{1}^{2}}\left(1+\frac{3}{2} \frac{1}{\varrho_{1}^{2}}\right)\right], \\
& \varrho_{1}=\frac{r_{1}}{2 \mathcal{R}_{g}},
\end{aligned}
$$

and $\theta_{1}$ is the angle between $\mathbf{r}_{1}$ and the axis of revolution as in Eq. (3.35). We note the limits

$$
\begin{gathered}
\lambda \rightarrow\left(\frac{3}{r_{1}^{3}}\left[1-\frac{8}{15 \sqrt{\pi}} \varrho_{1}^{5}+\cdots\right], \frac{1}{2 \sqrt{\pi} \mathcal{R}_{g}^{3}} e^{-\varrho_{1}^{2}}\right) \\
\text { for }\left(r_{1} \ll \mathcal{R}_{g}, r_{1} \gg \mathcal{R}_{g}\right) .
\end{gathered}
$$

Since $B_{\mathrm{VII}}$ is (positive, negative) for the (prolate, oblate) cases, Eq. (3.46) implies that $\mathcal{E}_{E}$ in these cases is (smaller, larger) on the axis of revolution with $\theta_{1}=0$ than in the plane $\theta_{1}=\pi / 2$ perpendicular to the axis and intersecting it in the center of the particle. Thus $\mathcal{E}_{E}$ is smaller along those directions $\theta_{1}$ for which the particle surface is further away from the particle center. Since $\mathcal{E}_{E}$ vanishes on the particle surface, this is a plausible result.

Equations (3.45) and (3.46), which apply for $l, s$ $\ll r_{1}, \mathcal{R}_{g}$, can be compared with the expressions

$$
\mathcal{E}_{E}\left(L=\infty ; \mathbf{r}_{1}\right)=\left(1-\frac{Q_{0}\left(\xi_{1}\right)}{Q_{0}(\xi)}, 1-\frac{Q_{0}\left(i \xi_{1}\right)}{Q_{0}(i \xi)}\right)
$$

for the (prolate, oblate) cases, which apply for $l, s, r_{1} \ll \mathcal{R}_{g}$. Equations (3.51) follow since the finite limit on the left-hand side due to (2.2) satisfies the Laplace equation, and $\left(Q_{0}\left(\xi_{1}\right), Q_{0}\left(i \xi_{1}\right) / i\right)$ with $\xi_{1}$ defined in Eqs. (3.21) and (3.24) are indeed solutions of the Laplace equation which vanish for $\xi_{1} \rightarrow \infty$ and have constant values $\left(Q_{0}(\xi)\right.$, $\left.Q_{0}(i \xi) / i\right)$ on the surface $\xi_{1}=\xi$ of the (prolate, oblate) ellipsoid. A more explicit form of $\mathcal{E}_{E}\left(\infty ; \mathbf{r}_{1}\right)$ follows by inserting Eqs. (3.10) into (3.51), see also Ref. 12. In particular for $E$ equal to the circular disk with radius $R_{\text {disk }}=f$,

$$
\mathcal{E}_{\text {disk }}\left(\infty ;\left(0, z_{1}\right)\right)=1-\frac{2}{\pi} \arctan \frac{f}{z_{1}}
$$

along the axis of revolution and

$$
\mathcal{E}_{\text {disk }}\left(\infty ;\left(r_{1, \perp}, 0\right)\right)=1-\frac{2}{\pi} \arcsin \frac{f}{r_{1, \perp}}
$$

along the radial direction within the plane of the disk. These expressions also determine the bulk normalized monomer density $\mathcal{M}$, since $\mathcal{M}_{E}=\mathcal{E}_{E}^{2}$ for $\mathcal{R}_{g} \rightarrow \infty$. Expanding (3.51) for $l, s \ll r_{1}$ with the help of (3.34) and using the expressions (3.7) and (3.9) for the amplitudes $B_{\mathrm{I}}$ and $B_{\mathrm{VII}}$, one obtains

$$
\begin{gathered}
\mathcal{E}_{E}\left(\infty ; \mathbf{r}_{1}\right)=1-\frac{B_{\mathrm{I}} f}{4 \pi r_{1}}+\frac{B_{\mathrm{VII}} f^{3}}{8 \pi r_{1}^{3}}\left(1-3 \cos ^{2} \theta_{1}\right) ; \\
l, s \ll r_{1} .
\end{gathered}
$$

This is consistent with the leading isotropic and anisotropic contributions (3.45) and (3.46) in the regime $l, s \ll r_{1} \ll \mathcal{R}_{g}$, for which both types of expressions are valid.

We defer a discussion of the free energy cost $\delta_{E} F$ to immerse the ellipsoid in the polymer solution to the next subsection.

\section{B. Interaction between an ellipsoid and a wall}

The evaluation of the free energy $\left(\delta_{E} F\right)_{W}$ required to immerse the small ellipsoid with its center at a distance $z_{E}$ from a planar wall proceeds as in Sec. II B. First consider the leading contribution

$$
\begin{aligned}
{\left[\left(\delta_{E} F\right)_{W}\right]_{\text {leading }} / p_{0} } & =f B_{\mathrm{I}} \mathcal{L}\left[\chi_{h}\left(z_{E}\right)\right]^{2} \\
& =f B_{\mathrm{I}} \mathcal{R}_{g}^{2} \mathcal{M}_{h}\left(z_{E} / \mathcal{R}_{g}\right),
\end{aligned}
$$

which is of first order in the particle size and arises from the isotropic operator $O_{\mathrm{I}}$. The right-hand side of (3.55) equals the contribution $R g_{1}$ in (2.38) except that $R A_{\mathrm{I}} \rightarrow f B_{\mathrm{I}}$ and $\mathcal{M}_{h}$ is the monomer density in the half space of Eq. (2.51), with $z_{S}$ replaced by $z_{E}$.

The free energy cost $\left[\delta_{E} F\right]_{\text {leading }}$ to immerse the small ellipsoid in unbounded bulk is given by (3.55) with $\mathcal{M}_{h}$ $=1$. Explicit results for the special cases of a weakly deformed sphere, a needle, and a circular disk follow by substituting the expressions for $f B_{\text {I }}$ from Eqs. (3.11), (3.14), and (3.17). Note that the free energy cost for a disk is smaller (by a factor of $2 / \pi$ ) than for a sphere with the same radius, as expected since the polymer depletion is weaker in the former case. A small prolate ellipsoid in a solution in unbounded bulk was considered in Ref. 12 where $f B_{\text {I }}$ was denoted by $8 \pi E$.

The leading anisotropic contribution to $\left(\delta_{E} F\right)_{W}$ arises from the two anisotropic operators $O_{\mathrm{VI}}$ and $O_{\mathrm{VII}}$. The calculation is similar to that of the term $g_{3, \mathrm{IV}}$ in Eq. (2.45) and leads to

$$
\begin{aligned}
{\left[\left(\delta_{E} F\right)_{W}\right]_{\text {leading anisotropic }} / p_{0}=} & f^{3} \mathcal{L}\left\{B_{\mathrm{VI}}\left[\partial_{\|} \chi_{h}(z)\right]^{2}\right. \\
& \left.+B_{\mathrm{VII}} \chi_{h}(z)\left[\partial_{\|}^{2} \chi_{h}(z)\right]\right\}_{z=z_{E}} \\
= & \left(\cos ^{2} \vartheta\right) f^{3} \mathcal{A}\left(z_{E} / \mathcal{R}_{g}\right)
\end{aligned}
$$

with $\chi_{h}$ given in (2.48), and

$$
\mathcal{A}=\left(B_{\mathrm{VI}}+B_{\mathrm{VII}}\right) \operatorname{erfc} \frac{z_{E}}{\mathcal{R}_{g}}-B_{\mathrm{VII}} \operatorname{erfc} \frac{z_{E}}{2 \mathcal{R}_{g}} .
$$

Here $\vartheta$ is the angle between the axis of revolution of the ellipsoid and the surface normal of the wall at $z=0$, as shown in Fig. 4. The factor $\cos \vartheta$ arises from the derivative $\partial_{\|}$defined in (3.5) via $\partial_{\|} \psi(z)=\rho_{z} \psi^{\prime}(z)$, with $\rho_{z}=\cos \vartheta$ the component of $\vec{\rho}$ perpendicular to the wall.

The function $\mathcal{A}$ has the limits

$$
\begin{aligned}
\mathcal{A}\left(z_{E} / \mathcal{R}_{g}\right) \rightarrow & \left\{B_{\mathrm{VI}}\left[1-\left(2+\frac{B_{\mathrm{VII}}}{B_{\mathrm{VI}}}\right) \frac{z_{E}}{\sqrt{\pi} \mathcal{R}_{g}}\right],\right. \\
& \left.-B_{\mathrm{VII}} \frac{2 \mathcal{R}_{g}}{\sqrt{\pi} z_{E}} e^{-\left(z_{E} / 2 \mathcal{R}_{g}\right)^{2}}\right\},
\end{aligned}
$$

for $\left(z_{E} / \mathcal{R}_{g} \ll 1, z_{E} / \mathcal{R}_{g} \gg 1\right)$, and in the intermediate regime changes sign at a scaled distance $z_{E} / \mathcal{R}_{g}=\zeta_{0}(l / s)$ which depends on the aspect ratio $l / s$. For the special case of a circular disk with a radius $R_{\text {disk }}=f$, the quantity

$$
\mathcal{A}=\mathcal{A}_{\text {disk }} \equiv 8\left(-\operatorname{erfc} \frac{z_{E}}{\mathcal{R}_{g}}+\frac{1}{3} \operatorname{erfc} \frac{z_{E}}{2 \mathcal{R}_{g}}\right)
$$

is shown in Fig. 5. 


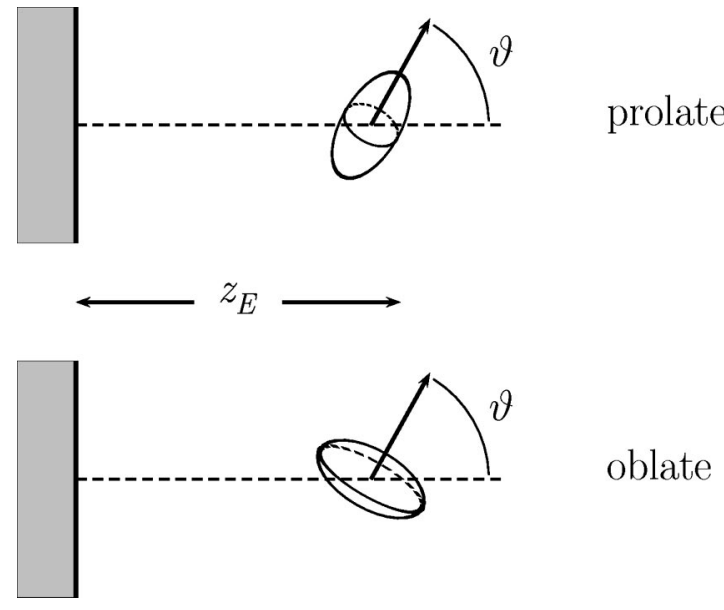

FIG. 4. Particles of prolate and oblate ellipsoidal shape near a planar wall. The ellipsoid is oriented parallel to the wall for $\vartheta=\pi / 2$ in the prolate case and for $\vartheta=0$ in the oblate case.

Since $B_{\mathrm{VI}}$ and $B_{\mathrm{VII}}$ are both (positive, negative) for (prolate, oblate) ellipsoids, the free energy $\left(\delta_{E} F\right)_{W}$ for larger particle-wall separations $z_{E} / \mathcal{R}_{g}>\zeta_{0}$ is lowest if the ellipsoid is aligned perpendicular to the wall with $\vartheta=(0, \pi / 2)$, and for the smaller separations $z_{E} / \mathcal{R}_{g}<\zeta_{0}$ if it is aligned parallel to the wall with $\vartheta=(\pi / 2,0)$.

This behavior is suggested by the following crude argument: First consider the situation without a small particle and replace the bulk-normalized monomer density profile in the half space $\mathcal{M}_{h}$ with its point of inflection by a step function $\theta\left(z-\widetilde{\zeta}_{0} \mathcal{R}_{g}\right)$ which jumps from 0 to 1 on increasing the distance $z$ from the wall beyond a certain value $\widetilde{\zeta}_{0} \mathcal{R}_{g}$. Then insert a small uniaxially anisotropic particle such as a rod of length $l$ and align it parallel to the wall. If the center of the

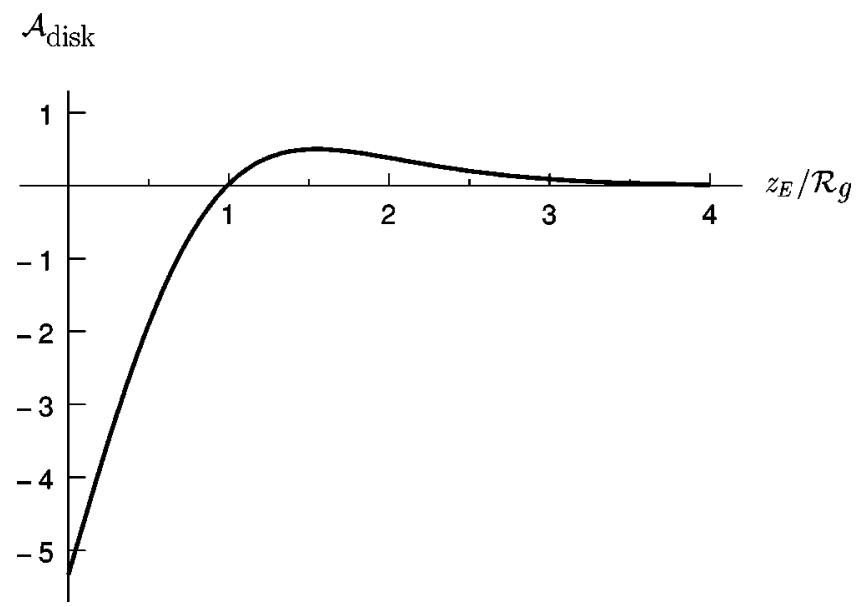

FIG. 5. The amplitude $\mathcal{A}$ which specifies the dependence (3.56) of the free energy of immersion $\left(\delta_{E} F\right)_{W}$ on the orientation of the small ellipsoidal particle with respect to the planar wall. The case $\mathcal{A}=\mathcal{A}_{\text {disk }}$ of a circular disk of radius $R_{\text {disk }}=f$ with $\mathcal{A}_{\text {disk }}$ given in Eq. (3.59) is shown. On decreasing the distance $z_{E}$ between the center of the disk and the wall, $\mathcal{A}_{\text {disk }}$ passes through a maximum value of 0.501 at $z_{E} / \mathcal{R}_{g}=1.55$, changes sign at $z_{E} / \mathcal{R}_{g}$ $=\zeta_{0, \text { disk }}=0.99$, and drops to a value of $-16 / 3$ for $z_{E} / \mathcal{R}_{g} \ll 1$. For $z_{E} / \mathcal{R}_{g}$ $>\zeta_{0 \text {,disk }}$ and $<\zeta_{0 \text {,disk }}$ the most favorable orientation of the disk is perpendicular and parallel to the wall with $\cos \vartheta=0$ and 1, respectively, see Eq. (3.56) and Fig. 4. For a general prolate or oblate small ellipsoid the qualitative form of $\mathcal{A}$ is that of $-\mathcal{A}_{\text {disk }}$ or $\mathcal{A}_{\text {disk }}$, respectively. rod is at a distance from the wall that is larger than $\widetilde{\zeta}_{0} \mathcal{R}_{g}$ (but smaller than $\widetilde{\zeta}_{0} \mathcal{R}_{g}+l / 2$ ), it is advantageous to turn the rod around its center perpendicular to the wall since then a part of the rod enters the region $z<\widetilde{\zeta}_{0} \mathcal{R}_{g}$ which is already polymer depleted. Conversely, if the distance of the center of the rod from the wall is smaller than $\widetilde{\zeta}_{0} \mathcal{R}_{g}$ (but larger than $\widetilde{\zeta}_{0} \mathcal{R}_{g}-l / 2$ ), turning the rod perpendicular to the wall is unfavourable, since then a part of the rod enters the region $z$ $>\widetilde{\zeta}_{0} \mathcal{R}_{g}$ which is not depleted by the wall.

\section{SUMMARY AND CONCLUDING REMARKS}

We have considered polymer depletion effects for mesoscopic colloidal particles with a size much smaller than the mesoscopic polymer lengths, such as the polymer radius of gyration $\mathcal{R}_{g}$. Extending the small particle expansion which applies in this "protein limit" beyond the leading order has enabled us to evaluate the orientation-dependent effective depletion interaction, mediated by a solution of free nonadsorbing ideal polymer chains, between a small ellipsoidal particle and a wall. Effects beyond leading order in the interaction between a spherical particle and a wall and between two spherical particles have also been discussed. Here is a summary of our main results:

For ideal polymers interacting with a small spherical particle in three dimensions, we have derived the small radius expansion up to third order in the particle radius. Up to this order the perturbation of the polymer system due to the particle corresponds to the five local operators given in Eq. (2.12) of the Gaussian Ginzburg-Landau field theory that is the "magnetic analogue" ${ }^{24}$ of the polymer system. The operators are multiplied by the particle radius raised to the scaling dimensions (2.13) or (2.23) and by the universal amplitudes (2.14) or (2.24), which are independent of the particular lattice or off-lattice realization of the random-walk like ideal polymer chains and of distant perturbations such as a wall or other particles. For quantities involving an integration over the polymer end positions, such as the end density profile (2.17) or the free energy (2.18) it costs to immerse the particle, there is a contact-term [see Eqs. (2.19)-(2.21)], in third order, with amplitude (2.22), that is also universal and independent of distant perturbations.

This expansion not only reproduces known results ${ }^{15,34,35}$ for the polymer partition function, the density profile of chain ends, and the free energy of immersion for the case of a single spherical particle, see Sec. II A, but also allows us to predict the form of the interactions between a particle and a wall (Sec. II B) and between two particles (Sec. II C).

Our analytical results $(2.38),(2.52)-(2.55)$ for the nextto-leading and next-to-next-to-leading contributions to the particle-wall interaction show an interesting dependence on the particle-wall distance and are in excellent agreement with a direct numerical calculation based on the multiple scattering method of Ref. 17, see Figs. 2 and 3.

We have checked that the new results (2.52), (2.53), and (2.65) for the polymer-induced interactions between a sphere and a wall and between two spheres beyond leading order in the sphere radius $R$ show for $\mathcal{R}_{g}$ much larger than the 
sphere-wall and sphere-sphere separations the correct limiting behaviors that are known from Refs. 17 and 16, compare the discussions near Eq. (2.57) and at the end of Sec. II C.

The small particle expansion can be generalized to anisotropic particles by including anisotropic derivativeoperators. For a small particle of prolate or oblate ellipsoidal shape with large axis $l$ and small axis $s$, as in Fig. 1, the leading operators are the two given in Eq. (3.3). It is convenient to use half of the interfocal distance as the length unit for the particle size [see Eq. (3.6)]. The corresponding universal amplitudes, which depend on the aspect ratio $l / s$ of the ellipsoid, are given in Eqs. (3.7)-(3.9) for the leading isotropic and anisotropic operators, with limiting forms for a weakly deformed sphere, a needle, and a circular disk in Eqs. (3.11)-(3.19).

For a single ellipsoid the expansion leads to the new results (3.45)-(3.49) for the leading isotropic and anisotropic parts of the end-density profile. These are valid for distances $r_{1}$ from the ellipsoid center satisfying $l, s \ll r_{1}, \mathcal{R}_{g}$ and correctly merge with the results (3.51)-(3.53) for the region $l, s, r_{1} \ll \mathcal{R}_{g}$ for which the diffusion equation (2.2) of the random-walk like ideal polymers ${ }^{24}$ reduces to Laplace's equation.

For a small ellipsoid at a distance $z_{E}$ from a planar wall, we obtain the leading contribution (3.55) to the particle-wall interaction, which is of first order in the size and independent of the orientation of the particle, and the leading dependence on orientation given by Eqs. (3.56) and (3.57). The latter is of third order in the particle size and depends on the angle $\vartheta$ between the axis of revolution of the ellipsoid and the surface normal of the wall, shown in Fig. 4, in the form of $\cos ^{2} \vartheta$ multiplied by a function $\mathcal{A}\left(z_{E} / \mathcal{R}_{g}\right)$ which has different signs for $z_{E} / \mathcal{R}_{g} \gg 1$ and $z_{E} / \mathcal{R}_{g} \ll 1$. For a disk the function $\mathcal{A}$ is shown in Fig. 5. The lowest free energy of interaction is attained on aligning the prolate or oblate ellipsoid perpendicular to the wall for the larger particle-wall distances $z_{E} / \mathcal{R}_{g}>\zeta_{0}$ and parallel to the wall for the smaller distances $z_{E} / \mathcal{R}_{g}<\zeta_{0}$, where $\zeta_{0}$ depends on the aspect ratio $l / s$.

The case of a small particle considered here should be compared with that of a large particle, in which the characteristic length scale of the embedding solution (such as $\mathcal{R}_{g}$ in case of a dilute polymer solution) is much smaller than the particle size. An interesting example of the latter case, studied recently in Ref. 39, consists of a rodlike particle close to a wall in a solution of colloidal hard spheres, with a size of the order of the width of the rod and much smaller than its length. As discussed above, for a small needle the change in the most favorable particle orientation from perpendicular to parallel to the wall on decreasing the particle-wall distance occurs at a distance of the order of $\mathcal{R}_{g}$, which is much larger than the size of the needle, i.e., when the needle is still far from the wall. In the large particle case the change takes place when the rod is already touching the wall at one end.

It would be interesting to generalize our work to include excluded volume interactions between chain-monomers and to study the case of an anisotropic particle in a critical fluid mixture. Computer simulations of anisotropic particles of arbitrary size in solutions of flexible polymers would be valuable.

\section{ACKNOWLEDGMENT}

The authors thank T. W. Burkhardt for useful discussions.

${ }^{1}$ R. Verma, J. C. Crocker, T. C. Lubensky, and A. G. Yodh, Phys. Rev. Lett. 81, 4004 (1998)

${ }^{2}$ Y. N. Ohshima et al., Phys. Rev. Lett. 78, 3963 (1997).

${ }^{3}$ D. Rudhardt, C. Bechinger, and P. Leiderer, Phys. Rev. Lett. 81, 1330 (1998).

${ }^{4}$ S. Wang, J. A. P. P. van Dijk, T. Odijk, and J. A. M. Smit, Biomacromolecules 2, 1080 (2001).

${ }^{5}$ A. M. Kulkarni, A. P. Chatterjee, K. S. Schweizer, and C. F. Zukoski, J. Chem. Phys. 113, 9863 (2002).

${ }^{6}$ O. Annunziata, N. Asherie, A. Lomakin, J. Pande, O. Ogun, and G. B. Benedek, Proc. Natl. Acad. Sci. U.S.A. 99, 14165 (2002).

${ }^{7}$ S. Asakura and F. Oosawa, J. Chem. Phys. 22, 155 (1954); J. Polym. Sci. 33, 183 (1958).

${ }^{8}$ M. A. Bates and D. Frenkel, Phys. Rev. E 62, 5225 (2000).

${ }^{9}$ F. M. van der Kooij, M. Vogel, and H. N. W. Lekkerkerker, Phys. Rev. E 62, 5397 (2000)

${ }^{10}$ P. G. de Gennes, C. R. Acad. Sc. Paris B 288, 359 (1979).

${ }^{11}$ T. Odijk, Macromolecules 29, 1842 (1996).

${ }^{12}$ T. Odijk, Physica A 278, 347 (2000).

${ }^{13}$ P. G. Bolhuis, E. J. Meijer, and A. A. Louis, cond-mat/0210528.

${ }^{14}$ A. Milchev and A. Bhattacharya, J. Chem. Phys. 117, 5415 (2002).

${ }^{15}$ E. Eisenriegler, A. Hanke, and S. Dietrich, Phys. Rev. E 54, 1134 (1996).

${ }^{16}$ A. Hanke, E. Eisenriegler, and S. Dietrich, Phys. Rev. B 59, 6853 (1999).

${ }^{17}$ A. Bringer, E. Eisenriegler, F. Schlesener, and A. Hanke, Eur. Phys. J. B 11, 101 (1999).

${ }^{18}$ E. Eisenriegler, J. Chem. Phys. 113, 5091 (2000).

${ }^{19}$ See, e.g., C.G. Callan in Methods in Field Theory, edited by R. Balian and J. Zinn-Justin, Session 26, (North-Holland, Amsterdam, 1976), pp. 41-77.

${ }^{20}$ T. Burkhardt and E. Eisenriegler, Phys. Rev. Lett. 74, 3189 (1995).

${ }^{21}$ E. Eisenriegler and U. Ritschel, Phys. Rev. B 51, 13717 (1995).

${ }^{22}$ A. Hanke, F. Schlesener, E. Eisenriegler, and S. Dietrich, Phys. Rev. Lett. 81, 1885 (1998).

${ }^{23}$ F. Schlesener, A. Hanke, and S. Dietrich, J. Stat. Phys. 110, 981 (2003).

${ }^{24}$ P. G. de Gennes, Scaling Concepts in Polymer Physics (Cornell University Press, Ithaca, 1979).

${ }^{25}$ J. des Cloizeaux and G. Jannink, Polymers in Solution (Clarendon, Oxford, 1990).

${ }^{26}$ L. Schäfer, Excluded Volume Effects in Polymer Solutions (Springer, Heidelberg, 1998).

${ }^{27}$ E. Eisenriegler, Polymer Near Surfaces (World Scientific, Singapore, 1993); E. Eisenriegler, in Field Theoretical Tools in Polymer- and Particle-Physics, edited by $\mathrm{H}$. Meyer-Ortmanns and A. Klümper, Lecture Notes in Physics (Springer, Berlin, 1998), Vol. 508.

${ }^{28} \mathrm{~K}$. Binder, in Phase Transitions and Critical Phenomena, edited by C. Domb and J. L. Lebowitz (Academic, London, 1983), Vol. 8.

${ }^{29} \mathrm{H}$. W. Diehl, in Phase Transitions and Critical Phenomena, edited by C. Domb and J. L. Lebowitz (Academic, London, 1986), Vol. 10; H. W. Diehl, Int. J. Mod. Phys. B 11, 3503 (1997).

${ }^{30}$ Approximating the density profile of chain monomers in the presence of two particles by the product of the profiles of the two single particles, the interaction between two spheres induced by ideal nonadsorbing polymer chains has been estimated for arbitrary size ratio in R. Tuinier, G. A. Vliegenthart, and H. N. W. Lekkerkerker, J. Chem. Phys. 113, 10768 (2000).

${ }^{31}$ M. Abramowitz and I. A. Stegun, Handbook of Mathematical Functions (Dover, New York, 1972).

${ }^{32}$ D. Amit, Field Theory, the Renormalization Group, and Critical Phenomena (McGraw-Hill, New York, 1978); J. Zinn-Justin, Quantum Field Theory and Critical Phenomena (Clarendon, Oxford, 1989).

${ }^{33}$ The single-particle related bulk-averages $\left\langle\varphi_{1,2} \cdot O_{j}\right\rangle_{\text {bulk }}$ for $j=\mathrm{III}$ and $\mathrm{V}$ have different structures, except for $d=3$ since $\langle\Phi(0) \Phi(0)\rangle_{\text {bulk }}^{2} \propto t$ in $d$ $=3$. However, the two corresponding half-space averages $\left\langle\varphi_{1,2} \cdot O_{j}\right\rangle_{h}$, 
which are related to a particle near a wall, have different structures, even in $d=3$.

${ }^{34}$ R. Lipowsky, Europhys. Lett. 30, 197 (1995).

${ }^{35}$ K. M. Jansons and C. G. Phillips, J. Colloid Interface Sci. 137, 75 (1990).

${ }^{36}$ The factor 5 on the right side of Eq. (2.57) is the sum of the contributions

4 and 1 which arise from the operators $O_{\mathrm{IV}}$ and $O_{\mathrm{V}}$, respectively.
${ }^{37}$ C. Flammer, Spheroidal Wave Functions (Stanford University Press, Stanford, California, 1957).

${ }^{38}$ The angles $\varphi_{k}$ in the $x-y$ plane between $\mathbf{r}_{k, \perp}$ and the $x$ axis should not be confused with the product $\varphi_{1,2}$ of Ginzburg-Landau fields in Eq. (2.7).

${ }^{39}$ R. Roth, R. van Roij, D. Andrienko, K. R. Mecke, and S. Dietrich, Phys. Rev. Lett. 89, 088301 (2002). 